\title{
On the Application of Intersection Theory to Feynman Integrals: the Multivariate Case
}

\author{
Federico Gasparotto $^{a, b}$ and Manoj K. Mandal ${ }^{b, a}$ \\ ${ }^{a}$ Dipartimento di Fisica e Astronomia, Università di Padova, \\ Via Marzolo 8, 35131 Padova, Italy \\ ${ }^{b}$ INFN, Sezione di Padova, \\ Via Marzolo 8, 35131 Padova, Italyy \\ E-mail: federico.gasparotto@studenti.unipd.it, manojkumar.mandal@pd.infn.it
}

This is a contribution to the proceedings of the MathemAmplitudes 2019 conference held in December 2019 in Padova, Italy and it is built upon the series of papers [1-4].

In the framework of intersection theory a direct projection of any given integral in terms of a preferred basis is directly achieved, thus avoiding the traditional linear system solving procedure. The coefficients of the decomposition are expressed in terms of intersection numbers. In this contribution we review their derivation, focusing on the recursive algorithm presented in $[3,5]$, applicable to integrals admitting a multivariate representation.

This proceedings is complementary to the one by Frellesvig and Mattiazzi [6] concerning the univariate case. For other contributions see also [7-12].

MathemAmplitudes 2019: Intersection Theory \& Feynman Integrals Padova, Italy

18-20 December 2019 


\section{Introduction}

Feynman integrals (FIs) are ubiquitous in the computation of multi-loop scattering amplitudes within the realm of Quantum Field Theory. Typically, at two-loop level, the number of integrals appearing is of the order $O\left(10^{5}\right)$. The computation of these integrals is performed in two stages: i) the reduction to a smaller set of independent integrals, known as Master Integrals (MIs) and ii) the evaluation of these independent MIs. Focusing on i), it is known that FIs in dimensional regularization are related by integration-by-parts identities (IBPs) [13]. Thanks to IBPs it is possible to build a huge linear system of equations among FIs; solving this system, essentially via Gauss' elimination, the vast majority of FIs is expressed as a linear combination of fewer basic integrals the above-mentioned MIs [14]. However, as the number of loops, external particles and masses increase, the algebraic manipulations required become very challenging and demanding.

Recently, a novel framework [1], relying on twisted de Rham co-homology and intersection theory, was proposed in order to describe relations among FIs. An underlying vector space for the FIs was identified; it was shown that this vector space is endowed with a scalar product, the so called intersection number. Thanks to these ideas, any FI can be decomposed into a basis of MIs, resembling the decomposition of a generic vector in the basis of a vector space. This approach avoided the generation of intermediate, auxiliary expressions, needed for applying Gauss' elimination in case of the standard IBP-based approaches. In [2], this idea was extensively applied to a number of FIs supported on maximal cuts, thus admitting a one-fold integral representation, thanks to the use of uni-variate intersection numbers. Then this proposal was further extended for generic FIs [3, 4], admitting multi-fold integral representations, through the evaluation of multi-variate intersection numbers, via a recursive algorithm proposed in [5]. Evaluation of multivariate intersection numbers in the case of logarithmic differential forms have been previously addressed in [15-23]. The study of intersection numbers in the context of FIs as well as their optimization is currently ongoing [24-26] - see [8, 9, 27, 28] for review articles; for the special application to canonical differential equations [29] see [30, 31].

In these proceedings, we review the evaluation of multivariate intersection numbers, required to obtain a direct decomposition of any given FI in terms of MIs, as well as the differential equation satisfied by them [32-34]. This contribution is organized as follows. First, in sec. 2, we describe the basic notations of intersection theory, and we introduce the twisted co-homology group, which is nothing but the above-mentioned vector space for FIs. Then in sec. 3, we explain in detail the ingredients needed to compute the (multivariate) intersection numbers, touching upon the 1-variable case (see [6] for more details), then generalizing it to 2-variable and eventually to $n$-variable cases. Then in sec. 4 , we show the application of multivariate intersection numbers to obtain the differential equation obeyed by the maximally cut two-loop double-box MIs. Finally, in sec. 5 we discuss the conclusions and further outlooks.

\section{Integral Decomposition and Intersection Theory}

We will consider generalized hypergeometric integrals of the following form

$$
I=\int_{C_{R}^{(\mathbf{n})}} u(\mathbf{z}) \varphi_{L}^{(\mathbf{n})}(\mathbf{z})
$$

where: $u(\mathbf{z})=\prod_{i} \mathcal{B}_{i}^{\gamma_{i}}$ is a multivalued function; $C_{R}^{(\mathbf{n})}$ is an integration domain such that $\prod_{i} \mathcal{B}_{i}\left(\partial C_{R}^{(\mathbf{n})}\right)=0$; $\varphi_{L}^{(\mathbf{n})}(\mathbf{z})$ is a single-valued differential $n$-form. In the context of the FIs discussed in these proceedings, we consider $u(\mathbf{z})=\mathcal{B}(\mathbf{z})^{\gamma}$, where $\mathcal{B}$ is the so called Baikov polynomial [35]. The exponent $\gamma$ reads $\gamma=(d-\ell-E-1) / 2$, where $d$ denotes the space time dimensions, $\ell$ the number of loops and $E$ the number of independent external 
momenta. $\varphi_{L}^{(\mathbf{n})}(\mathbf{z})$ has the following form

$$
\varphi_{L}^{(\mathbf{n})}(\mathbf{z})=\hat{\varphi}_{L}^{(\mathbf{n})}(\mathbf{z}) d^{n} \mathbf{z}, \quad \hat{\varphi}_{L}^{(\mathbf{n})}(\mathbf{z})=\frac{f(\mathbf{z})}{z_{1}^{a_{1}} \ldots z_{n}^{a_{n}}},
$$

where $f(\mathbf{z})$ is a rational function and $a_{i}$ are integer exponents ${ }^{1}$.

Using the key property $\mathcal{B}\left(\partial C_{R}^{(\mathbf{n})}\right)=0$ and employing Stokes' theorem, we infer

$$
0=\int_{C_{R}^{(\mathbf{n})}} d\left(u \xi_{L}^{(\mathbf{n}-\mathbf{1})}\right)=\int_{C_{R}^{(\mathbf{n})}}\left(d u \wedge \xi_{L}^{(\mathbf{n}-\mathbf{1})}+u d \xi_{L}^{(\mathbf{n}-\mathbf{1})}\right)=\int_{C_{R}^{(\mathbf{n})}} u\left(\frac{d u}{u} \wedge+d\right) \xi_{L}^{(\mathbf{n}-\mathbf{1})}=\int_{C_{R}^{(\mathbf{n})}} u \nabla_{\omega} \xi_{L}^{(\mathbf{n}-\mathbf{1})}
$$

where $\xi_{L}^{(\mathbf{n}-\mathbf{1})}$ is an arbitrary $(n-1)$ differential form and

$$
\nabla_{\omega}=d+\omega \wedge, \quad \omega=d \log u=\sum_{i=1}^{n} \hat{\omega}_{i} d z_{i} .
$$

Now, we can define an equivalence class among differential $n$-forms $\varphi_{L}^{(\mathbf{n})}$, namely

$$
\varphi_{L}^{(\mathbf{n})} \sim \varphi_{L}^{(\mathbf{n})}+\nabla_{\omega} \xi_{L}^{(\mathbf{n}-\mathbf{1})}
$$

since they give the same result upon integration.

In other words, eq. (5) leads to consider the following

$$
H_{\omega}^{n}=\left\{n \text {-forms } \varphi_{L}^{(\mathbf{n})} \mid \nabla_{\omega} \varphi_{L}^{(\mathbf{n})}=0\right\} /\left\{\nabla_{\omega} \xi^{(\mathbf{n}-\mathbf{1})}\right\} .
$$

This is known as the twisted cohomology group and its elements are called twisted cocycles. We just mention that it is possible to introduce a similar structure for the integration contours, the so called twisted homology group, whose elements are denoted by $\left.\mid C_{R}^{(\mathbf{n})}\right]$. Within this framework, the integral $I$ in eq. (1) can be identified as a pairing between $\left\langle\varphi_{L}^{(\mathbf{n})}\right|$ and $\left.\mid C_{R}^{(\mathbf{n})}\right]$.

$$
\left.I=\left\langle\varphi_{L}^{(\mathbf{n})}\right| C_{R}^{(\mathbf{n})}\right] .
$$

For us, the study of integrals of the form $(1,7)$ reduces to the study of the space in eq. (6): it can be considered the vector space of FIs.

On the other hand, we can also define a dual vector space, the so called dual twisted cohomology group: $\left(H_{\omega}^{n}\right)^{*}=H_{-\omega}^{n}$ where $\nabla_{-\omega}$ now replaces $\nabla \omega$ in eq. (6); the importance of this auxiliary structure will be clear in a moment.

We will denote the dimension of the (dual) twisted cohomology group, or, in other words, the number of MIs, by $v$; thanks to complex Morse (Picard-Lefschetz) theory, $v$ is determined as the number of critical points of the function $\log u(\mathbf{z})$ [36]

$$
\begin{aligned}
v & =\operatorname{dim}\left(H_{ \pm \omega}^{n}\right) \\
& =\text { nr. solutions of: } \quad \hat{\omega}_{i}=\partial_{z_{i}} \log u(\mathbf{z})=0 \quad 1 \leq i \leq n .
\end{aligned}
$$

We will denote a set of basis and dual basis of differential forms as $\left\langle e_{i}^{(\mathbf{n})}\right| \in H_{\omega}^{n}$ and $\left|h_{i}^{(\mathbf{n})}\right\rangle \in H_{-\omega}^{n}$ with $i=1, \ldots, v$ respectively.

${ }^{1}$ Additionally, we assume that all the poles present in $\varphi_{L}^{(\mathbf{n})}$ are regulated by $u(\mathbf{z})$. When, in the context of FIs, this assumption is often violated we refer the interested reader to [1-4] for the description of the necessary regularization procedure. 
Following [1-3], the decomposition of an arbitrary integral in terms of MIs, or, better, of an arbitrary form, say $\left\langle\varphi_{L}^{(\mathbf{n})}\right|$ in terms of master forms $\left\langle e_{i}^{(\mathbf{n})}\right|$ reads

$$
\left\langle\varphi_{L}^{(\mathbf{n})}\right|=\sum_{i=1}^{v} c_{L, i}\left\langle e_{i}^{(\mathbf{n})}\right| .
$$

The key point is that it is possible to introduce a bilinear non-degenerate pairing, the so called intersection number, denoted by $\left\langle\phi_{L}^{(\mathbf{n})} \mid \phi_{R}^{(\mathbf{n})}\right\rangle$ with $\left\langle\phi_{L}^{(\mathbf{n})}\right| \in H_{\omega}^{n}$ and $\left|\phi_{R}^{(\mathbf{n})}\right\rangle \in H_{-\omega}^{n}$; it can be thought as the scalar product in our vector space.

Once the intersection number is introduced, it can be shown that the coefficients in eq. (9) read [1-4]

$$
c_{L, i}=\sum_{j=1}^{v}\left\langle\varphi_{L}^{(\mathbf{n})} \mid h_{j}^{(\mathbf{n})}\right\rangle\left(\mathbf{C}^{-1}\right)_{j i}
$$

with

$$
\mathbf{C}_{i j}=\left\langle e_{i}^{(\mathbf{n})} \mid h_{j}^{(\mathbf{n})}\right\rangle \quad 1 \leq i, j \leq v .
$$

So the coefficients of the decomposition can be directly achieved expressed in terms of intersection numbers. We notice that it is possible to decompose a dual form, say $\left|\varphi_{R}^{(\mathbf{n})}\right\rangle$ in terms of the dual basis

$$
\left|\varphi_{R}^{(\mathbf{n})}\right\rangle=\sum_{i=1}^{v} c_{R, i}\left|h_{i}^{(\mathbf{n})}\right\rangle
$$

with

$$
c_{R, i}=\sum_{i=1}^{v}\left(\mathbf{C}^{-1}\right)_{i j}\left\langle e_{j}^{(\mathbf{n})} \mid \varphi_{R}^{(\mathbf{n})}\right\rangle .
$$

Even if eqs. $(12,13)$ do not have a direct physical interpretation they will be useful in our construction.

The main goal of the next section in this contribution is to review the evaluation of the intersection number in the multivariate case.

\section{Multivariate Intersection Numbers}

As anticipated above, we discuss here a recursive algorithm for the evaluation of the multivariate intersection numbers introduced in [5], and applied, for the first time, to FIs in [3, 4]. Loosely speaking the idea of the recursive method consists in assuming $(n-1)$-variable intersection numbers known and computable, and then expressing the $n$-variable intersection numbers in terms of those. Here, we will first discuss the univariate intersection number and then show explicitly how we can use this knowledge to evaluate a 2-variable intersection number. Eventually, this can be generalized to obtain the expression of a $n$-variable intersection number.

\subsection{1-variable intersection number}

Let us consider the integral in eq. (1) depending on 1 variable, dubbed as $z_{1}$, namely

$$
\left.I=\int_{C_{R}^{(1)}} u\left(z_{1}\right) \varphi_{L}^{(\mathbf{1})}\left(z_{1}\right)=\left\langle\varphi_{L}^{(\mathbf{1})}\right| C_{R}^{(\mathbf{1})}\right] .
$$

Rewriting eq. (3) for one variable, we get

$$
0=\int_{C_{R}^{(\mathbf{1})}} d_{z_{1}}(u \xi)=\int_{\mathcal{C}_{R}^{(\mathbf{1})}} u\left(d_{z_{1}} \xi+d_{z_{1}} \log u \wedge \xi\right)=\int_{\mathcal{C}_{R}^{(1)}} u \nabla_{\omega_{1}} \xi
$$


where

$$
\nabla_{\omega_{1}}=d_{z_{1}}+\omega_{1} \wedge, \quad \omega_{1}=d_{z_{1}} \log u, \quad \xi \text { any } 0 \text {-form } .
$$

So the equivalence relation among differential 1 -forms reads

$$
\varphi_{L}^{(\mathbf{1})} \sim \varphi_{L}^{(\mathbf{1})}+\nabla_{\omega_{1}} \xi
$$

since they give the same result upon integration, for any 0 -form $\xi .^{2}$

One might imagine defining the univariate intersection pairing $\left\langle\varphi_{L}^{(\mathbf{1})} \mid \varphi_{R}^{(\mathbf{1})}\right\rangle$ as

$$
\left\langle\varphi_{L}^{(\mathbf{1})} \mid \varphi_{R}^{(\mathbf{1})}\right\rangle \stackrel{?}{=} \frac{1}{2 \pi i} \int_{X_{1}} \varphi_{L}^{(\mathbf{1})} \wedge \varphi_{R}^{(\mathbf{1})} .
$$

As discussed in [8], eq. (18) is ill defined; it can be seen as a " $0 / 0$ problem", since, on the one hand, $\varphi_{L}^{(\mathbf{1})} \wedge \varphi_{R}^{(\mathbf{1})}$ vanishes at the integrand level (being $d z_{1} \wedge d z_{1}=0$ ) and, on the other hand the poles in the differential forms yield a divergent integral. The solution consists in considering the compact supported version of $\varphi_{L}^{(\mathbf{1})}$ laying in the same equivalence class, namely $\iota_{\omega_{1}}\left(\varphi_{L}^{(\mathbf{1})}\right)$ [16]. So eq. (18) becomes

$$
\left\langle\varphi_{L}^{(\mathbf{1})} \mid \varphi_{R}^{(\mathbf{1})}\right\rangle=\frac{1}{2 \pi i} \int_{X_{1}} \iota_{\omega_{1}}\left(\varphi_{L}^{(\mathbf{1})}\right) \wedge \varphi_{R}^{(\mathbf{1})}
$$

The explicit realization of the regularization is

$$
\iota_{\omega_{1}}\left(\varphi_{L}^{(\mathbf{1})}\right)=\varphi_{L}^{(\mathbf{1})}-\sum_{p \in \mathcal{P}_{\omega_{1}}} \nabla_{\omega_{1}}\left(h_{p}\left(z_{1}, \bar{z}_{1}\right) \psi_{i}^{(p)}\right)
$$

$h_{p}\left(z_{1}, \bar{z}_{1}\right)$ is a complex bump function, see Fig. (1); $\psi_{i}^{(p)}$ is the holomorphic local solution of the differential equation

$$
\nabla_{\omega_{1}} \psi_{i}^{(p)}=\varphi_{L}^{(1)},
$$

around every pole $p$ of $\omega_{1}$ (including $\infty$ ), whose set is denoted by $\mathcal{P}_{\omega_{1}}$. Thanks to Stokes' theorem it can be

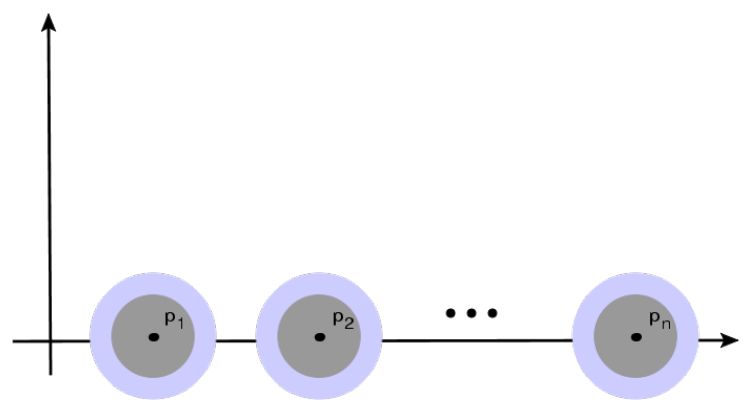

Figure 1: The bump functions $h_{p}\left(z_{1} \bar{z}_{1}\right)$; they are equal to 1 in the dark grey area, they decay smoothly in the light grey are and they vanish outside - white area.

shown that eq. (19) localizes to

$$
\left\langle\varphi_{L}^{(\mathbf{1})} \mid \varphi_{R}^{(\mathbf{1})}\right\rangle=\sum_{p \in \mathcal{P}_{\omega_{1}}} \operatorname{Res}_{z_{1}=p}\left(\psi_{i}^{(p)} \varphi_{R}^{(\mathbf{1})}\right) .
$$

${ }^{2}$ For ease of notation, from now on we will denote 0 -forms as $\boldsymbol{\xi}=\boldsymbol{\xi}^{(\mathbf{0})}$. 


\subsection{2-variable intersection number}

Now, we consider the integral in eq. (1) depending on two integration variables $\left\{z_{1}, z_{2}\right\}$,

$$
\left.I=\int_{C_{R}^{(2)}} u\left(z_{1}, z_{2}\right) \varphi_{L}^{(2)}\left(z_{1}, z_{2}\right)=\left\langle\varphi_{L}^{(2)}\right| C_{R}^{(2)}\right],
$$

where $\mathbf{2}=\{1,2\}, \varphi_{L}^{(\mathbf{2})}$ is a differential 2-form depending in $z_{1}$ and $z_{2}$ and the $C_{R}^{(\mathbf{2})}$ denotes a two-dimensional integration region embedded in an ambient space $X$, having complex dimension 2 . Now, we consider the fibration of the space $X$ into one-dimensional spaces $X_{2} \ni z_{2}$ and $X_{1} \ni z_{1} .{ }^{3}$ The former is dubbed as outer space, while the latter is the inner space. Eq. (8) allows us to determine $v_{1}$, the number of (dual) basis elements in the inner space. Their explicit choices are denoted by $\left\langle e_{i}^{(\mathbf{1})}\right|$ and $\left|h_{i}^{(\mathbf{1})}\right\rangle$ for $i=1, \ldots, v_{1}$ for the basis and dual basis respectively. Since the 1 -variable intersection numbers are known at this stage, we can write the following decompositions

$$
\begin{aligned}
& \left\langle\varphi_{L}^{(\mathbf{2})}\right|=\sum_{i=1}^{v_{1}}\left\langle e_{i}^{(\mathbf{1})}\right| \wedge\left\langle\varphi_{L, i}^{(2)}\right|, \\
& \left|\varphi_{R}^{(2)}\right\rangle=\sum_{i=1}^{\nu_{1}}\left|h_{i}^{(\mathbf{1})}\right\rangle \wedge\left|\varphi_{R, i}^{(2)}\right\rangle ;
\end{aligned}
$$

$\left\langle\varphi_{L, i}^{(2)}\right|$ and $\left|\varphi_{R, i}^{(2)}\right\rangle$ can be considered as the coefficients of the decompositions, and, in general will depend on the variable $z_{2}$ (but not on $z_{1}$ ). Benefiting from eqs. $(24,25)$, one might imagine defining the 2 -variable intersection number as

$$
\begin{aligned}
\left\langle\varphi_{L}^{(\mathbf{2})} \mid \varphi_{R}^{(2)}\right\rangle & \stackrel{?}{=} \frac{1}{(2 \pi i)^{2}} \int_{X} \varphi_{L}^{(2)} \wedge \varphi_{R}^{(2)} \\
& \stackrel{?}{=} \sum_{i, j=1}^{\nu_{1}} \frac{1}{2 \pi i} \int_{X_{2}} \varphi_{L, i}^{(2)} \wedge \varphi_{R, j}^{(2)} \times \frac{1}{2 \pi i} \int_{X_{1}} \iota_{\omega_{1}}\left(e_{i}^{(\mathbf{1})}\right) \wedge h_{j}^{(\mathbf{1})}
\end{aligned}
$$

Once again eq. (26) needs to be regularized. Denoting by $\iota_{\mathbf{\Omega}^{(2)}}\left(\varphi_{L, i}^{(2)}\right)$ the regularized version ${ }^{4}$ of $\varphi_{L, i}^{(2)}$, eq. (26) becomes

$$
\left\langle\varphi_{L}^{(\mathbf{2})} \mid \varphi_{R}^{(\mathbf{2})}\right\rangle=\sum_{i, j=1}^{\nu_{1}} \frac{1}{2 \pi i} \int_{X_{2}} \iota_{\mathbf{\Omega}^{(2)}}\left(\varphi_{L, i}^{(2)}\right) \wedge \varphi_{R, j}^{(2)} \times \frac{1}{2 \pi i} \int_{X_{1}} \iota_{\omega_{1}}\left(e_{i}^{(\mathbf{1})}\right) \wedge h_{j}^{(\mathbf{1})} .
$$

The innermost integral is nothing but 1 -variable intersection number among (dual) inner basis elements. We will denote

Therefore we have

$$
\left(\mathbf{C}_{(1)}\right)_{i j}=\left\langle e_{i}^{(\mathbf{1})} \mid h_{j}^{(\mathbf{1})}\right\rangle \quad \text { (see also eq. (11)) }
$$

$$
\left\langle\varphi_{L}^{(2)} \mid \varphi_{R}^{(2)}\right\rangle=\sum_{i, j=1}^{\nu_{1}} \frac{1}{2 \pi i} \int_{X_{2}} \iota_{\mathbf{\Omega}^{(2)}}\left(\varphi_{L, i}^{(2)}\right) \wedge \varphi_{R, j}^{(2)} \times\left(\mathbf{C}_{(1)}\right)_{i j}
$$

In order to build $\iota_{\mathbf{\Omega}^{(2)}}\left(\varphi_{L, i}^{(2)}\right)$ explicitly, we have first to generalize eq. (15) in the case of an integral over $\left(z_{1}, z_{2}\right)$. Let us reconsider eq. (23) together with eq. (24)

$$
\int_{C_{R}^{(2)}} u \varphi_{L}^{(2)}=\sum_{i=1}^{v_{1}} \int_{C_{R}^{(2)}} \varphi_{L, i}^{(2)} \int_{C_{R}^{(1)}} u e_{i}^{(\mathbf{1})}=\sum_{i=1}^{\nu_{1}} \int_{C_{R}^{(2)}} \varphi_{L, i}^{(2)} u_{i}
$$

\footnotetext{
${ }^{3}$ This fibration does not necessarily signify that $X=X_{2} \times X_{1}$, since $X_{1}=X_{1}\left(z_{1}, z_{2}\right)$ can depend on $z_{2}$ (but $X_{2}$ does not depend on $\left.z_{1}\right)$; for more detail see $[5,8]$ and references therein.

${ }^{4}$ The notation will be clear in a moment.
} 
where

$$
u_{i}=\int_{\mathcal{C}_{R}^{(1)}} u e_{i}^{(1)}
$$

So eq. (15) becomes

$$
\begin{aligned}
0=\sum_{i=1}^{v_{1}} \int_{C_{R}^{(2)}} d_{z_{2}}\left(u_{i} \xi_{i}\right) & =\sum_{i=1}^{v_{1}} \int_{C_{R}^{(2)}}\left(u_{i} d_{z_{2}} \xi_{i}+d_{z_{2}} u_{i} \wedge \xi_{i}\right) \\
& =\sum_{i, j=1}^{v_{1}} \int_{\mathcal{C}_{R}^{(2)}} u_{j}\left(d_{z_{2}} \delta_{j i}+\boldsymbol{\Omega}_{i j}^{(2)} \wedge\right) \xi_{i} \\
& =\sum_{i, j=1}^{\nu_{1}} \int_{\mathcal{C}_{R}^{(2)}} u_{j}\left(\nabla_{\mathbf{\Omega}^{(2)}}\right)_{j i} \xi_{i},
\end{aligned}
$$

where

$$
\left(\nabla_{\mathbf{\Omega}^{(2)}}\right)_{j i}=d_{z_{2}} \delta_{j i}+\boldsymbol{\Omega}_{i j}^{(2)} \wedge
$$

and $\boldsymbol{\Omega}_{i j}^{(2)}$ is implicitly defined by

$$
d_{z_{2}} u_{i}=\boldsymbol{\Omega}_{i j}^{(2)} u_{j}
$$

Recalling the explicit expression (31), we infer that $\boldsymbol{\Omega}_{i j}^{(2)}$ is nothing but the matrix controlling the system of differential equations fulfilled by the internal bases elements $e_{i}^{(\mathbf{1})}$; its elements can be expressed in terms of univariate intersection numbers

$$
\mathbf{\Omega}_{i j}^{(2)}=\left\langle\left(\partial_{z_{2}}+\omega_{2}\right) e_{i}^{(\mathbf{1})} \mid h_{k}^{(\mathbf{1})}\right\rangle\left(\mathbf{C}_{(\mathbf{1})}^{-1}\right)_{k j}, \quad\left(\mathbf{C}_{(\mathbf{1})}\right)_{k j}=\left\langle e_{k}^{(\mathbf{1})} \mid h_{j}^{(\mathbf{1})}\right\rangle
$$

Therefore the equivalence relations for $\varphi_{L, i}^{(2)}$ are given by

$$
\varphi_{L, i}^{(2)} \sim \varphi_{L, i}^{(2)}+\left(\nabla_{\mathbf{\Omega}^{(2)}}\right)_{i j} \xi_{j}
$$

Having understood how to build equivalence relations for $\varphi_{L, i}^{(2)}$, mimicking eq. (20), we have

$$
\iota_{\mathbf{\Omega}^{(2)}}\left(\varphi_{L, i}^{(2)}\right)=\varphi_{L, i}^{(2)}-\sum_{q \in \mathcal{P}_{\mathbf{\Omega}^{(2)}}} \nabla_{\mathbf{\Omega}^{(2)} i j}\left(h_{q}\left(z_{2}, \bar{z}_{2}\right) \psi_{j}^{(q)}\right)
$$

now $\psi_{j}^{(q)}$ is the local holomorphic solution of the system of differential equation

$$
\nabla_{\mathbf{\Omega}^{(2)} i j} \psi_{j}^{(q)}=\varphi_{L, i}^{(2)}
$$

around every pole $q$ of $\boldsymbol{\Omega}^{(2)}$ (including $\infty$ ), whose set is denoted by $\mathcal{P}_{\mathbf{\Omega}^{(2)}}$.

Given eq. (29) and the explicit expression of eq. (37), Stokes' theorem leads to

$$
\left\langle\varphi_{L}^{(\mathbf{2})} \mid \varphi_{R}^{(\mathbf{2})}\right\rangle=\sum_{i, j=1}^{v_{1}} \sum_{q \in \mathcal{P}_{\mathbf{\Omega}^{(2)}}} \operatorname{Res}_{z_{2}=q}\left[\psi_{i}^{(q)} \varphi_{R, j}^{(2)} \times\left(\mathbf{C}_{(\mathbf{1})}\right)_{i j}\right]
$$




\section{$3.3 n$-variable intersection number}

Eq. (39) can be generalized to $n$-variables intersection numbers, assuming $(n-1)$-variables intersection are computable and known; the explicit expression reads

$$
\left\langle\varphi_{L}^{(\mathbf{n})} \mid \varphi_{R}^{(\mathbf{n})}\right\rangle=\sum_{i, j=1}^{v_{n-1}} \sum_{q \in \mathcal{P}_{\mathbf{\Omega}^{(n-1)}}} \operatorname{Res}_{z_{n}=q}\left[\psi_{i}^{(q)}\left(\mathbf{C}_{(\mathbf{n}-\mathbf{1})}\right)_{i j} \varphi_{R, j}^{(n)}\right]
$$

where

$$
\begin{aligned}
\left(\mathbf{C}_{(\mathbf{n}-\mathbf{1})}\right)_{i j} & =\left\langle e_{i}^{(\mathbf{n}-\mathbf{1})} \mid h_{j}^{(\mathbf{n}-\mathbf{1})}\right\rangle \\
\varphi_{L, i}^{(n)} & =\left\langle\varphi_{L}^{(\mathbf{n})} \mid h_{j}^{(\mathbf{n}-\mathbf{1})}\right\rangle\left(\mathbf{C}_{(\mathbf{n}-\mathbf{1})}^{-1}\right)_{j i} \\
\varphi_{R, j}^{(n)} & =\left(\mathbf{C}_{(\mathbf{n}-\mathbf{1})}^{-1}\right)_{j k}\left\langle e_{k}^{(\mathbf{n}-\mathbf{1})} \mid \varphi_{R}^{(\mathbf{n})}\right\rangle
\end{aligned}
$$

and $\psi_{i}^{q}$ the local solution of

$$
\nabla_{\mathbf{\Omega}^{(n-1)} i j} \psi_{j}^{(q)}=\varphi_{L, i}^{(n)}
$$

with

$$
\boldsymbol{\Omega}_{i j}^{(n)}=\left\langle\left(\partial_{z_{n}}+\omega_{n}\right) e_{i}^{(\mathbf{n}-\mathbf{1})} \mid h_{k}^{(\mathbf{n}-\mathbf{1})}\right\rangle\left(\mathbf{C}_{(\mathbf{n}-\mathbf{1})}^{-1}\right)_{k j}
$$

around the set of poles $\mathcal{P}_{\mathbf{\Omega}^{(n-1)}}$ of $\boldsymbol{\Omega}^{(n-1)}$ (including $\infty$ ).

\section{Massless Double-Box on the Maximal Cut}

We present in this section the intersection numbers required for the derivation of the system of differential equations fulfilled by the MIs of the massless double-box integral family, depicted in Fig. (2), on the maximal cut. For concreteness we will consider differentiation with respect to the invariant $s$.

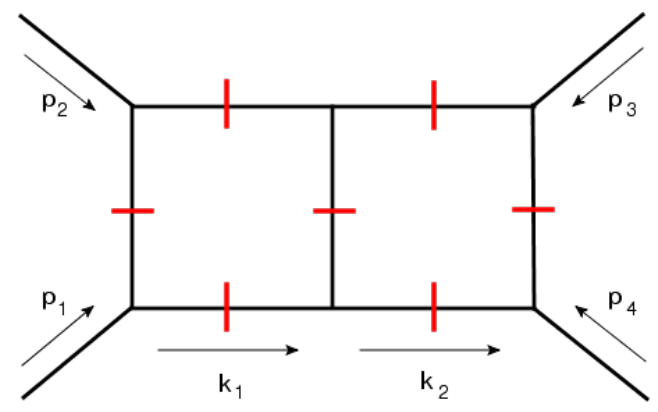

Figure 2: Massless double-box integral family; the external momenta are such that $p_{1}^{2}=p_{2}^{2}=p_{3}^{2}=p_{4}^{2}=0$ (with $\left.p_{4}=-p_{1}-p_{2}-p_{3}\right)$; the kinematic invariants read: $s=\left(p_{1}+p_{2}\right)^{2}$ and $t=\left(p_{2}+p_{3}\right)^{2}$.

The denominators are chosen as

$$
\begin{aligned}
& D_{1}=k_{1}^{2} \quad D_{2}=\left(k_{1}-p_{1}\right)^{2} \quad D_{3}=\left(k_{1}-p_{1}-p_{2}\right)^{2} \quad D_{4}=\left(k_{1}-k_{2}\right)^{2} \\
& D_{5}=\left(k_{2}-p_{1}-p_{2}\right)^{2} \quad D_{6}=\left(k_{2}-p_{1}-p_{2}-p_{3}\right)^{2} \quad D_{7}=k_{2}^{2}
\end{aligned}
$$


while the ISPs read

$$
D_{8} \equiv z_{1}=\left(k_{2}-p_{1}\right)^{2} \quad D_{9} \equiv z_{2}=\left(k_{1}-p_{1}-p_{2}-p_{3}\right)^{2} .
$$

On the maximal cut we are just left with an integral over $\left(z_{1}, z_{2}\right)$, and a given FI within the integral family is ${ }^{5}$

$$
\mathcal{I}_{a_{1}, \ldots, a_{7} ; a_{8}, a_{9}}^{\mathcal{K}}=\mathcal{K} \mathcal{I}_{a_{1}, \ldots, a_{7} ; a_{8}, a_{9}}
$$

with

$$
\mathcal{K}=(-s t(s+t))^{\frac{4-d}{2}} .
$$

In the language of intersection theory we have

$$
\mathcal{I}_{a_{1}, \ldots, a_{7} ; a_{8}, a_{9}}=\int_{C_{R}^{(2)}} u \varphi^{(2)}
$$

with

$$
u=\mathcal{B}^{\frac{d-6}{2}}, \quad \mathcal{B}=s z_{1} z_{2}\left(s\left(-t+z_{1}+z_{2}\right)+z_{1} z_{2}\right)
$$

Once the matrix of the system $\partial_{s} \mathcal{I}=\mathbf{\Omega} \mathcal{I}$ is known, the one for the system $\partial_{s} \mathcal{I}^{\mathcal{K}}=\mathbf{A} \mathcal{I}^{\mathcal{K}}$ follows trivially; the explicit expression is

$$
\mathbf{A}=\mathbf{\Omega}+\partial_{s} \log \mathcal{K} \mathbb{I}
$$

therefore we will focus on integrals of the form of eq. (50).

The counting of the critical points (see eq. (8)) reveals that there are $v=2$ MIs. They can be chosen as

$$
\left.\left.\mathcal{J}_{1}=\mathcal{I}_{1, \ldots, 1 ; 0,0}=\langle 1| C\right], \quad \mathcal{J}_{2}=\mathcal{I}_{1, \ldots, 1 ;-1,0}=\left\langle z_{1}\right| C\right]
$$

and the corresponding differential forms read

$$
\left\langle e_{1}^{(2)}\right|=\langle 1|, \quad\left\langle e_{2}^{(2)}\right|=\left\langle z_{1}\right| .
$$

We focus on the system of differential equation obeyed by the latter and, with respect to the invariant $s$. In order to do this, we identify the following differential forms

$$
\begin{aligned}
& \left\langle\Phi_{1}^{(2)}\right|=\partial_{s}\left\langle e_{1}^{(2)}\right|=\left\langle\left(\partial_{s}+\sigma\right) e_{1}^{(2)}\right| \\
& \left\langle\Phi_{2}^{(2)}\right|=\partial_{s}\left\langle e_{2}^{(2)}\right|=\left\langle\left(\partial_{s}+\sigma\right) e_{2}^{(2)}\right|
\end{aligned}
$$

with

$$
\sigma=d_{s} \log u=\frac{(d-6)\left(2 s\left(t-z_{1}-z_{2}\right)-z_{1} z_{2}\right)}{2 s\left(s\left(t-z_{1}-z_{2}\right)-z_{1} z_{2}\right)} .
$$

We can write the matrix $\boldsymbol{\Omega}$ in terms of intersection numbers (cf. eq. (10))

$$
\mathbf{\Omega}=\mathbf{F} \mathbf{C}^{-1}
$$

with

$$
\mathbf{F}_{i j}=\left\langle\Phi_{i}^{(2)} \mid e_{j}^{(2)}\right\rangle \quad \mathbf{C}_{i j}=\left\langle e_{i}^{(2)} \mid e_{j}^{(2)}\right\rangle
$$

Finally, in order to compute the intersection numbers in $\left(z_{1}, z_{2}\right)$, we have to specify the number of master forms, namely $v_{1}$, and their explicit expression, say $\left\langle\left. e_{i}^{(\mathbf{1})}\right|_{i=1, \ldots, v_{1}} \text { and } \mid h_{i}^{(\mathbf{1})}\right\rangle_{i=1, \ldots, v_{1}}$ with respect to the inner variable,

\footnotetext{
${ }^{5}$ We omit irrelevant $d$-dependent prefactors for our discussion.
} 
chosen to be $z_{1}$.

We find $v_{1}=1$, which amounts to the number of solutions of the equation $\omega_{1}=0$, and we choose

$$
\left\langle e^{(\mathbf{1})}\left|=\left\langle 1 / z_{1}|, \quad| h^{(\mathbf{1})}\right\rangle=\right| 1 / z_{1}\right\rangle .
$$

The explicit intersection numbers read

$$
\begin{aligned}
\mathbf{C}_{11}= & \frac{s^{2}\left(-3(3 d-20)(3 d-16) s^{2}-24(2(d-12) d+71) s t-4(5(d-12) d+177) t^{2}\right)}{16(d-7)^{2}(d-5)^{2}} \\
\mathbf{C}_{12}= & \frac{s^{2}\left(3(3 d-22)(3 d-20)(3 d-16) s^{3}+6(3 d-20)(d(9 d-113)+346) s^{2} t+8(d(11(d-19) d+1313)-2724) s t^{2}+8(d-7)(d-6)(d-5) t^{3}\right)}{32(d-8)(d-7)^{2}(d-5)^{2}} \\
\mathbf{C}_{21}= & \frac{s^{2}\left(3(3 d-20)(3 d-16)(3 d-14) s^{3}+6(3 d-16)(d(9 d-103)+286) s^{2} t+8(d(11(d-17) d+1049)-1944) s t^{2}+8(d-7)(d-6)(d-5) t^{3}\right)}{32(d-7)^{2}(d-5)^{2}(d-4)} \\
\mathbf{C}_{22}= & \frac{s^{3}\left(-3(3 d-22)(3 d-20)(3 d-16)(3 d-14) s^{3}-12(3 d-20)(3 d-16)(5(d-12) d+172) s^{2} t-24(3(d-12) d+104)(5(d-12) d+178) s t^{2}\right)}{64(d-8)(d-7)^{2}(d-5)^{2}(d-4)} \\
& -\frac{s^{3} t^{3}(d-6)^{2}}{(d-8)(d-7)(d-5)(d-4)} ;
\end{aligned}
$$

and

$$
\begin{aligned}
\mathbf{F}_{11}= & \frac{s\left(-3(3 d-20)(3 d-16)(5 d-26) s^{2}+12(d((294-17 d) d-1683)+3192) s t+4(d((296-17 d) d-1705)+3252) t^{2}\right)}{32(d-7)^{2}(d-5)^{2}} \\
\mathbf{F}_{12}= & \frac{s\left(3(3 d-22)(3 d-20)(3 d-16)(5 d-26) s^{3}+6(3 d-20)(d(d(39 d-695)+4078)-7896) s^{2} t\right)}{64(d-8)(d-7)^{2}(d-5)^{2}} \\
& +\frac{s\left((d(d(d(40 d-973)+8824)-35358)+52824) s t^{2}+(d-7)(d-6)(d-5)(3 d-16) t^{3}\right)}{8(d-8)(d-7)^{2}(d-5)^{2}} \\
\mathbf{F}_{21}= & \frac{s\left(3(3 d-20)(3 d-16)(3 d-14)(5 d-24) s^{3}+6(3 d-16)(d(13 d(3 d-49)+3422)-6064) s^{2} t\right)}{64(d-7)^{2}(d-5)^{2}(d-4)} \\
& +\frac{s\left((d(d(d(40 d-881)+7234)-26268)+35616) s t^{2}+(d-7)(d-6)(d-5)(3 d-16) t^{3}\right)}{8(d-7)^{2}(d-5)^{2}(d-4)} \\
\mathbf{F}_{22}= & \frac{s^{2}\left(-3(3 d-22)(3 d-20)(3 d-16)(3 d-14)(5 d-24) s^{3}-12(3 d-20)(3 d-16)(d(d(22 d-371)+2042)-3688) s^{2} t\right)}{128(d-8)(d-7)^{2}(d-5)^{2}(d-4)} \\
& -\frac{s^{2}\left(3(d(d(3 d(d(19 d-550)+6330)-108644)+309072)-34988) s t^{2}+2(d-7)(d-6)(d-5)(d(13 d-144)+400) t^{3}\right)}{16(d-8)(d-7)^{2}(d-5)^{2}(d-4)} .
\end{aligned}
$$

Therefore eq. (58) leads to

$$
\boldsymbol{\Omega}=\left(\begin{array}{cc}
\frac{2(d-6) s+(3 d-16) t}{2(s+t)} & -\frac{d-4}{s(s+t)} \\
-\frac{(d-4) t}{2(s+t)} & \frac{(5 d-24) s+(3 d-16) t}{2 s(s+t)}
\end{array}\right)
$$

and, finally, eq. (52) yields

$$
\mathbf{A}=\left(\begin{array}{cc}
\frac{(d-6) t-2 s}{s(s+t)} & -\frac{d-4}{s(s+t)} \\
-\frac{(d-4) t}{2(s+t)} & \frac{(3 d-16) s+2(d-6) t}{2 s(s+t)}
\end{array}\right)
$$

This is in agreement with LiteRed [37]

\section{Conclusion}

In these proceedings we reviewed the novel method [1-4] for the decomposition of FIs onto a basis of MIs within the realm of twisted de Rham (co)-homology and intersection theory. The required projection formulas $(10,13)$ are expressed in terms of intersection numbers. We described in details the evaluation of the multivariate intersection numbers following a recursive algorithm. Specifically, we showed how the concepts of the evaluation 
of univariate intersection numbers can be used to obtain the multivariate intersection numbers, recursively. We applied these ideas to derive the system of differential equations satisfied by the MIs appearing in the maximally cut massless double-box integral family.

Let us mention that, despite the success of the application of the method reviewed here to physically relevant examples [1-4], there is still much more to be said on the interplay between intersection theory and FIs. Recent developments include: development and optimization of the multivariate algorithm [24, 38], the application of the framework of twisted relative (co)-homology [25, 26, 39], the study of the $\epsilon$-factorized form of differential equations (even beyond polylogarithms) [10, 30, 31, 40] and the diagrammatic coaction for FIs [41, 42] just to mention a few.

\section{Acknowledgements}

We would like to thank Hjalte Frellesvig, Stefano Laporta, Pierpaolo Mastrolia, Luca Mattiazzi and Sebastian Mizera for their collaborations, as well as for discussions and explanations. Additionally, we thank Seva Chestnov and Henrik J. Munch for collaborating on closely related topics. Special thanks to all the participants of MathemAmplitudes 2019 for having created a sparkling and stimulating atmosphere.

The work of M.K.M is supported by Fellini - Fellowship for Innovation at INFN funded by the European Union's Horizon 2020 research and innovation programme under the Marie Skłodowska-Curie grant agreement No 754496. F.G. is supported by Fondazione Cassa di Risparmio di Padova e Rovigo - CARIPARO.

\section{References}

[1] P. Mastrolia and S. Mizera, Feynman Integrals and Intersection Theory, JHEP 02 (2019) 139, [arXiv: 1810.03818].

[2] H. Frellesvig, F. Gasparotto, S. Laporta, M. K. Mandal, P. Mastrolia, L. Mattiazzi, and S. Mizera, Decomposition of Feynman Integrals on the Maximal Cut by Intersection Numbers, JHEP 05 (2019) 153, [arXiv: 1901.11510].

[3] H. Frellesvig, F. Gasparotto, M. K. Mandal, P. Mastrolia, L. Mattiazzi, and S. Mizera, Vector Space of Feynman Integrals and Multivariate Intersection Numbers, Phys. Rev. Lett. 123 (2019), no. 20 201602, [arXiv: 1907.02000].

[4] H. Frellesvig, F. Gasparotto, S. Laporta, M. K. Mandal, P. Mastrolia, L. Mattiazzi, and S. Mizera, Decomposition of Feynman Integrals by Multivariate Intersection Numbers, arXiv: 2008.04823.

[5] S. Mizera, Aspects of Scattering Amplitudes and Moduli Space Localization, arXiv: 1906. 02099.

[6] H. Frellesvig and L. Mattiazzi, On the Application of Intersection Theory to Feynman Integrals: The Univariate Case, in MathemAmplitudes 2019: Intersection Theory and Feynman Integrals, 2, 2021. arXiv:2102.01576.

[7] P. Mastrolia, From Diagrammar to Diagrammalgebra, in MathemAmplitudes 2019: Intersection Theory and Feynman Integrals.

[8] S. Mizera, Status of Intersection Theory and Feynman Integrals, PoS MA2019 (2019) 016, [arXiv:2002.10476]. 
[9] S. Weinzierl, Applications of intersection numbers in physics, in MathemAmplitudes 2019: Intersection Theory and Feynman Integrals, 11, 2020. arXiv : 2011.02865.

[10] F. Brown and C. Duhr, A double integral of dlog forms which is not polylogarithmic, 6, 2020. arXiv:2006.09413.

[11] P. Vanhove and F. Zerbini, Building blocks of closed and open string amplitudes, in MathemAmplitudes 2019: Intersection Theory and Feynman Integrals, 7, 2020. arXiv:2007.08981.

[12] D. Bendle, J. Boehm, W. Decker, A. Georgoudis, F.-J. Pfreundt, M. Rahn, and Y. Zhang, Module Intersection for the Integration-by-Parts Reduction of Multi-Loop Feynman Integrals, in MathemAmplitudes 2019: Intersection Theory and Feynman Integrals, 10, 2020. arXiv:2010.06895.

[13] K. G. Chetyrkin and F. V. Tkachov, Integration by Parts: The Algorithm to Calculate beta Functions in 4 Loops, Nucl. Phys. B192 (1981) 159-204.

[14] S. Laporta, High precision calculation of multiloop Feynman integrals by difference equations, Int. J. Mod. Phys. A15 (2000) 5087-5159, [hep-ph/0102033].

[15] K. Matsumoto, Quadratic Identities for Hypergeometric Series of Type $(k, l)$, Kyushu Journal of Mathematics 48 (1994), no. 2 335-345.

[16] K. Matsumoto, Intersection numbers for logarithmic k-forms, Osaka J. Math. 35 (1998), no. 4 873-893.

[17] K. Ohara, Y. Sugiki, and N. Takayama, Quadratic Relations for Generalized Hypergeometric Functions ${ }_{p} F_{p-1}$, Funkcialaj Ekvacioj 46 (2003), no. 2 213-251.

[18] Y. Goto, Twisted Cycles and Twisted Period Relations for Lauricella's Hypergeometric Function $F_{C}$, International Journal of Mathematics 24 (2013), no. 12 1350094, [arXiv: 1308 . 5535].

[19] Y. Goto and K. Matsumoto, The monodromy representation and twisted period relations for Appell's hypergeometric function $F_{4}$, Nagoya Math. J. 217 (03, 2015) 61-94.

[20] Y. Goto, Twisted period relations for Lauricella's hypergeometric functions $F_{A}$, Osaka J. Math. 52 (07, 2015) 861-879.

[21] Y. Goto, Intersection Numbers and Twisted Period Relations for the Generalized Hypergeometric Function ${ }_{m+1} F_{m}$, Kyushu Journal of Mathematics 69 (2015), no. 1 203-217.

[22] S. Mizera, Scattering Amplitudes from Intersection Theory, Phys. Rev. Lett. 120 (2018), no. 14141602 , [arXiv: 1711.00469].

[23] S.-J. Matsubara-Heo and N. Takayama, An algorithm of computing cohomology intersection number of hypergeometric integrals, arXiv: 1904.01253.

[24] S. Weinzierl, On the computation of intersection numbers for twisted cocycles, arXiv:2002.01930.

[25] S. Caron-Huot and A. Pokraka, Duals of Feynman Integrals, I: Differential Equations, arXiv:2104.06898.

[26] S. Caron-Huot and A. Pokraka, Duals of Feynman Integrals, 2: Generalized Unitarity, arXiv: 2112.00055. 
[27] S. L. Cacciatori, M. Conti, and S. Trevisan, Co-homology of Differential Forms and Feynman diagrams, arXiv:2107.14721.

[28] S. Weinzierl, Feynman Integrals, arXiv:2201.03593.

[29] J. M. Henn, Multiloop integrals in dimensional regularization made simple, Phys.Rev.Lett. 110 (2013), no. 25 251601, [arXiv: 1304.1806].

[30] S. Mizera and A. Pokraka, From Infinity to Four Dimensions: Higher Residue Pairings and Feynman Integrals, arXiv: 1910.11852.

[31] J. Chen, X. Xu, and L. L. Yang, Constructing Canonical Feynman Integrals with Intersection Theory, arXiv:2008.03045.

[32] A. Kotikov, The Differential equation method: Evaluation of complicated Feynman integrals, hep-ph/0011316.

[33] E. Remiddi, Differential equations for Feynman graph amplitudes, Nuovo Cim. A110 (1997) 1435-1452, [hep-th/9711188].

[34] T. Gehrmann and E. Remiddi, Using differential equations to compute two loop box integrals, Nucl.Phys.Proc.Suppl. 89 (2000) 251-255, [hep-ph/0005232].

[35] P. A. Baikov, Explicit solutions of the multiloop integral recurrence relations and its application, Nucl. Instrum. Meth. A389 (1997) 347-349, [hep-ph/9611449].

[36] R. N. Lee and A. A. Pomeransky, Critical points and number of master integrals, JHEP 11 (2013) 165, [arXiv: 1308.6676].

[37] R. N. Lee, Presenting LiteRed: a tool for the Loop InTEgrals REDuction, arXiv: 1212 . 2685.

[38] S.-J. Matsubara-Heo and N. Takayama, An algorithm of computing cohomology intersection number of hypergeometric integrals, arXiv: 1904.01253.

[39] K. Matsumoto, Relative twisted homology and cohomology groups associated with Lauricella's $F_{D}$, arXiv: 1804.00366.

[40] H. Frellesvig, On Epsilon Factorized Differential Equations for Elliptic Feynman Integrals, arXiv:2110.07968.

[41] S. Abreu, R. Britto, C. Duhr, E. Gardi, and J. Matthew, From positive geometries to a coaction on hypergeometric functions, JHEP 02 (2020) 122, [arXiv: 1910.08358].

[42] S. Abreu, R. Britto, C. Duhr, E. Gardi, and J. Matthew, The diagrammatic coaction beyond one loop, JHEP 10 (2021) 131, [arXiv:2106.01280]. 